\title{
Time-Frequency System Builds and Timing Strategy Research of VHF Band Antenna Array
}

\author{
Junqing Liu1,2, Liang Dong1,3,4, Min Wang1,4, Shaojie Guo ${ }^{1,2}$ \\ ${ }^{1}$ Yunnan Astronomical Observatories, Chinese Academy of Sciences, Kunming, China \\ ${ }^{2}$ University of Chinese Academy of Sciences, Beijing, China \\ ${ }^{3}$ Information College, Yunnan University, Kunming, China \\ ${ }^{4}$ Yunnan University-Yunnan Astronomical Observatories Information Technology United Laboratory, Kunming, \\ China \\ Email: ljq@ynao.ac.cn
}

Received 20 January 2016; accepted 26 February 2016; published 2 March 2016

\begin{abstract}
VHF (Very High Frequency) band antenna array will receive analog signal from universe for storage after digital sampling and adding time scale, and then do the interference analysis of different sub-station digital signal. It requires the time-frequency system with high precision and low drifting. This paper explains a time-frequency system of VHF band antenna, which can produce standard $10 \mathrm{MHz}$ signal and clock signal needed by sampler, to ensure that two computers which sampling data has the same system time and the storage data has the accurate time scale, the system includes time comparison programme based on the GPS network timing two different sampling control computers. Timing strategy uses a time comparison software which based on the Labview graphical programming platform. This software captures the system time of two computers to analyze and determine the time deviation when the two computers occurs time offset, and then grant the GPS time of NTP server to the two computers through local area network in this time deviation. Final results show that this method can automatically calibrate the system time of the computers in the LAN, Precision Can Reach 0.1 s Orless.
\end{abstract}

\section{Keywords}

Antenna Array, GPS, Network Timing

\section{Introduction}

The VHF band antenna introduced in this study is used in receiving analog signal from universe for astro observation. It contains a wealth of information about radio burst of sun and planets in VHF band below $80 \mathrm{MHz}$ [1]. For the radio observation of celestial body out of solar system, VHF band is not a simple extrapolation of radio bands which we have used, and it is an important observation band because we haven't explored universe in this 
band. Many astronomical phenomena are observable only in VHF band, and already anticipated scientific exploration include: the CME and radio burst of the sun [2] [3], pulsar emission, AKR emission, the large-scale and high- resolution radio characteristics of Milky Way in HF-VHF band, Galactic HII Distribution Survey, frequency variation characteristics of transient celestial.etc. The rich astronomical phenomena and radiation of VHF band are described in Figure 1.

The observed sensitivity and resolution depends on the geometry and total receiving area of antenna array [4] [5]. As key technology research, we supposed to build two sub-stations in Yunnan observatories, Chinese Academy of Science and Chenggong campus of Yunnan University, Yunnan, China respectively. In Figure 2, two sub-stations are $22 \mathrm{~km}$ away from each other, each station has 16 antennas and every antenna receives two polarization signals. Forming beam after sampling the received analog signal, and send the digital signals from sub-stations to process center. The antenna can form multi-beam to observe different radio sources.

Antenna array consists of 32 antennas, the sampling rate is 200 MSPS (Million Samples Per Second) and every sample has 12 bits. It will obtain a huge amount of data if every antenna captures signal from two polarization direction. To avoid this situation, four antennas form a Tile as Figure 3. As the experience of LOFAR (Low-Frequency Array), LBA (Low frequency Band Antenna) and VLA [6] (Very Large Array), its only need sum the same polarization signals of four antennas and every Tile outputs two polarization signals, and then three-quarters of the data will be reduced. Antenna array arrangement will affect the back-end signal processing, the weight parameter of each antenna is determined according to the trajectory of radio source and antenna array arrangement [7] as Figure 4.

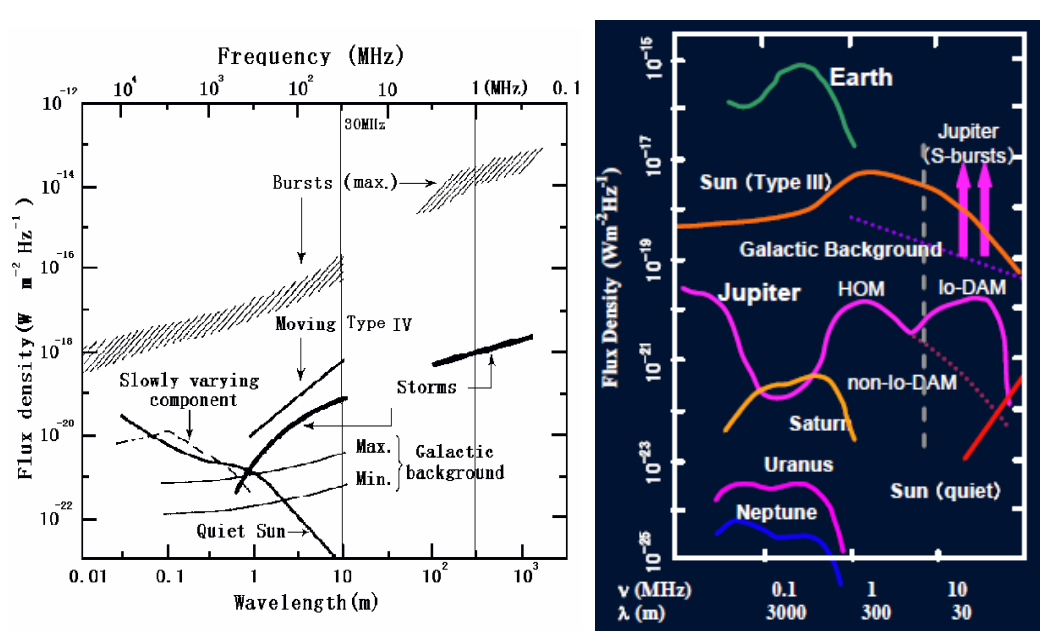

Figure 1. Radio traffic spectrum of VHF band, left: sun and the galactic, right: major planet.

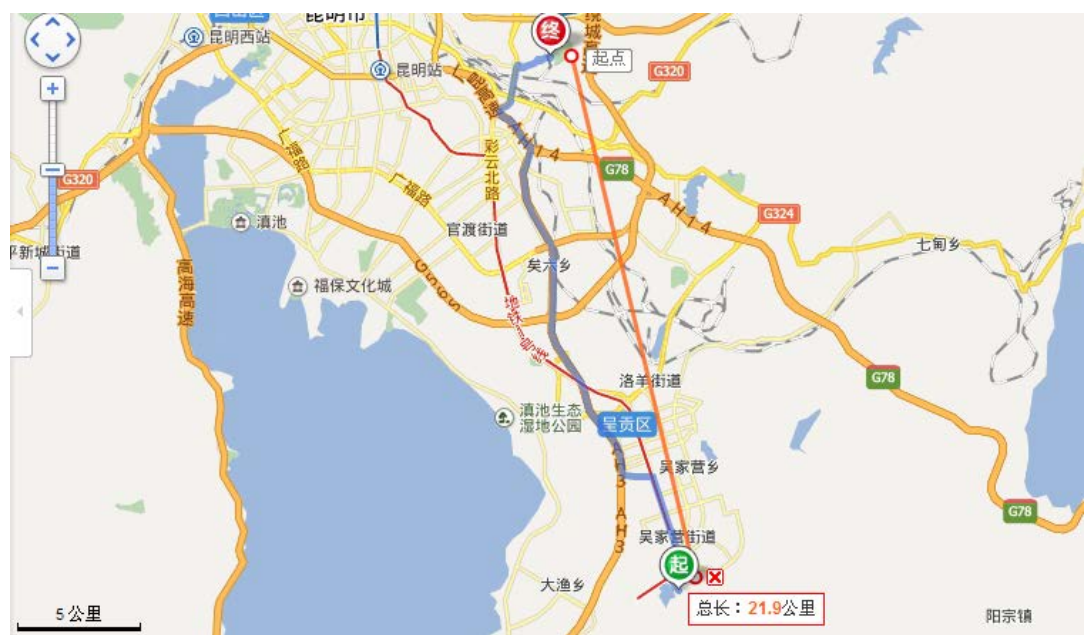

Figure 2. Two sub-stations away from 22 kilometers. 


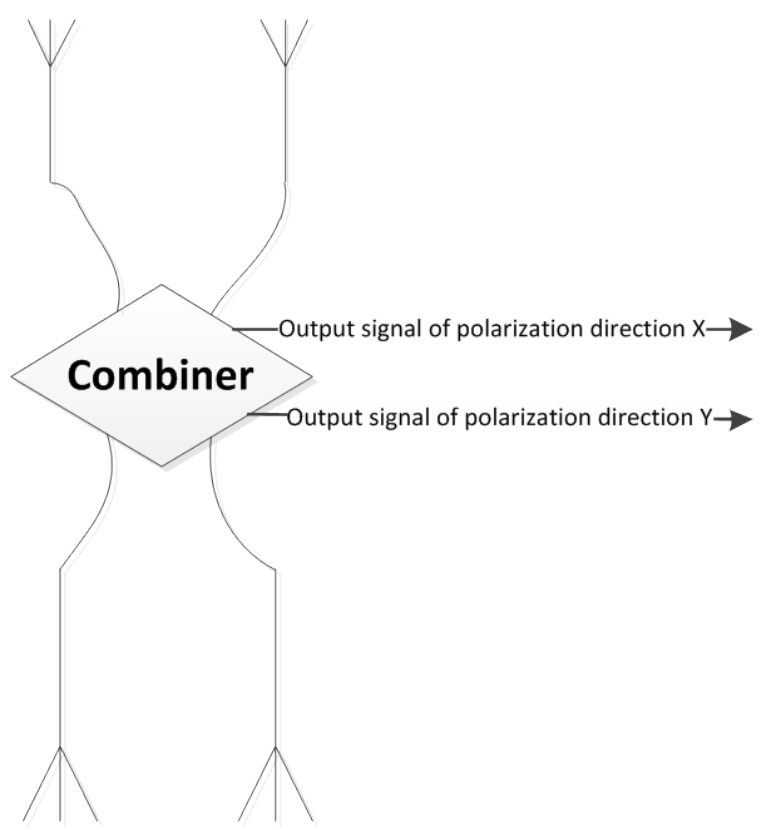

Figure 3. Spatial arrangement of a tile.

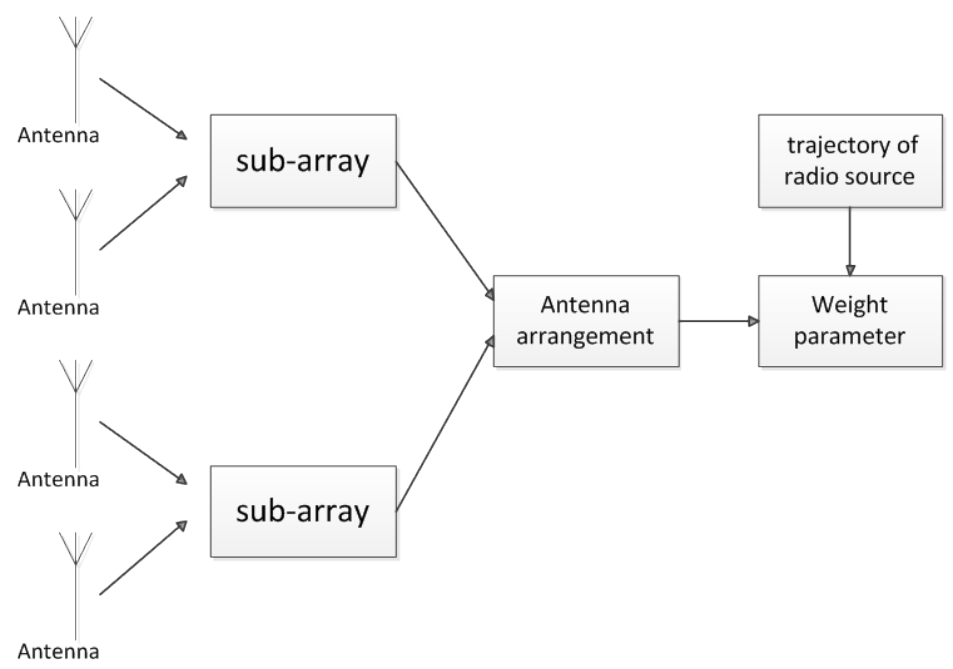

Figure 4. Schematic diagram of sub-array signal synthesis.

\section{Data Reception and Acquisition System}

The signals received by antennas will be filtered and amplified and then they are transmitted to the data acquisition board (AD6657). AD6657 has 200MPSP sample rate and four channel AD convertors, it connect with the FPGA signal processing board (HSC-ADC-EVALCZ).

Figure 5 describes the work pattern of AD6657 and HSC-ADC-EVALCZ. The analog signals received by antennas are transmitted to the AD6657 through four channel AD convertors [8], $200 \mathrm{MHz}$ clock signal will be induced into the board at the same time and the analog signal will be converted to digital signal to transmitted to the HSC-ADC-EVALCZ board [9]. HSC-ADC-EVALCZ connect computer through port USB2.0 and the corresponding software VisualAnalog can be used to compute data (FFT, integral etc.) and control data capture.

\section{Time-Frequency System of the Antenna array}

Time-frequency system is an important part of sub-stations and it provides reference frequency and time. Figure 6 


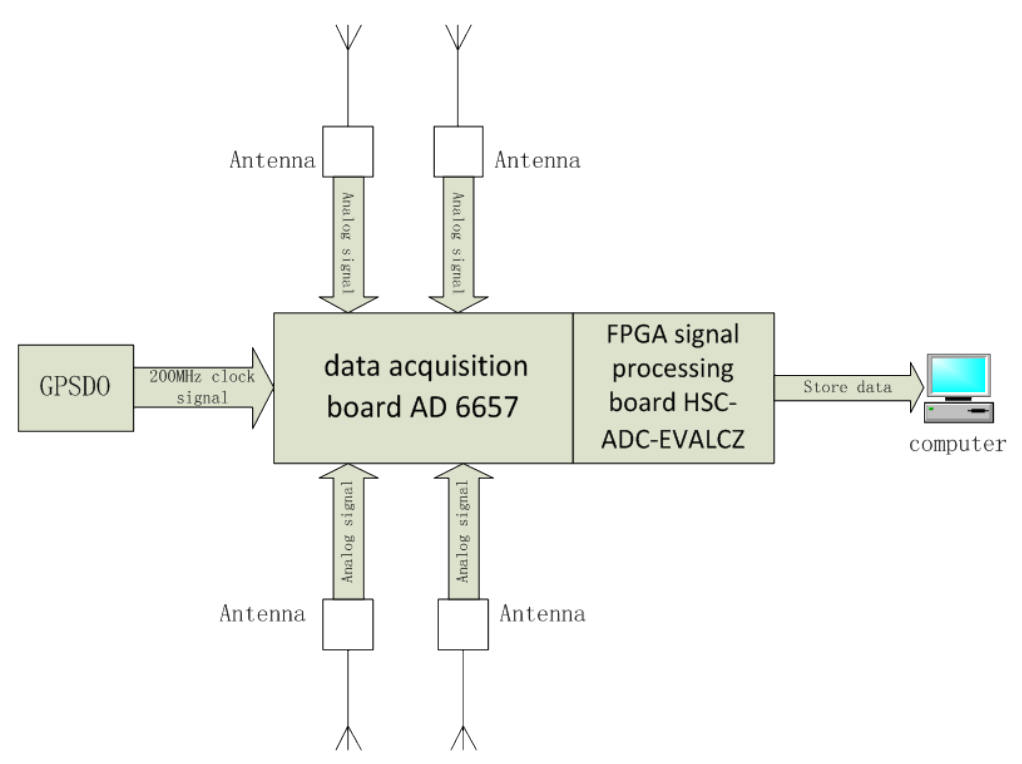

Figure 5. The acquisition system's block diagram.

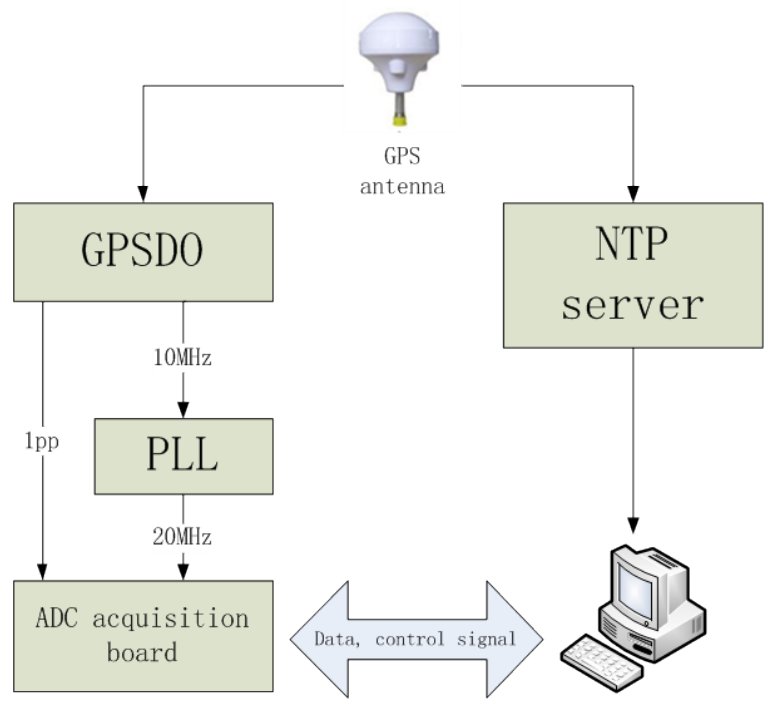

Figure 6. The time-frequency system architecture.

is the architecture of time-frequency system. Its functions mainly in the following aspects:

1) NTP server provides reference time to computer which control data sampling.

2) GPSDO generates a $10 \mathrm{MHz}$ signal and converted to $200 \mathrm{MHz}$ by PLL, as a precise external clock signal of AD6657.

As the clock signal of AD6657, it is required the phase errors caused by frequency instability less than 1 rad [10]. If $\sigma_{v}$ is the Allan deviation of clock signal, and there is a equation:

$$
2 \pi f \tau \sigma_{y} \leq 1 \mathrm{rad} \Rightarrow \sigma_{y} \leq \frac{1}{2 \pi f \tau}
$$

$f$ is $60 \mathrm{MHz}$ which is the center frequency the antenna array work on, $\tau$ is integration time. If $\tau=1 \mathrm{~s}$, it's need; $\sigma_{v} \leq 2.65 \times 10^{-9}$; if $\tau=100 \mathrm{~s}$, it's need $\sigma_{v} \leq 2.65 \times 10^{-11}$. The Allan deviation of the clock signal generated by GPSDO we used as Figure 7 and Table 1.

According to the allan deviation provided by Table 1, it is meet the need of observation when $\tau=800 \mathrm{~s}$. 


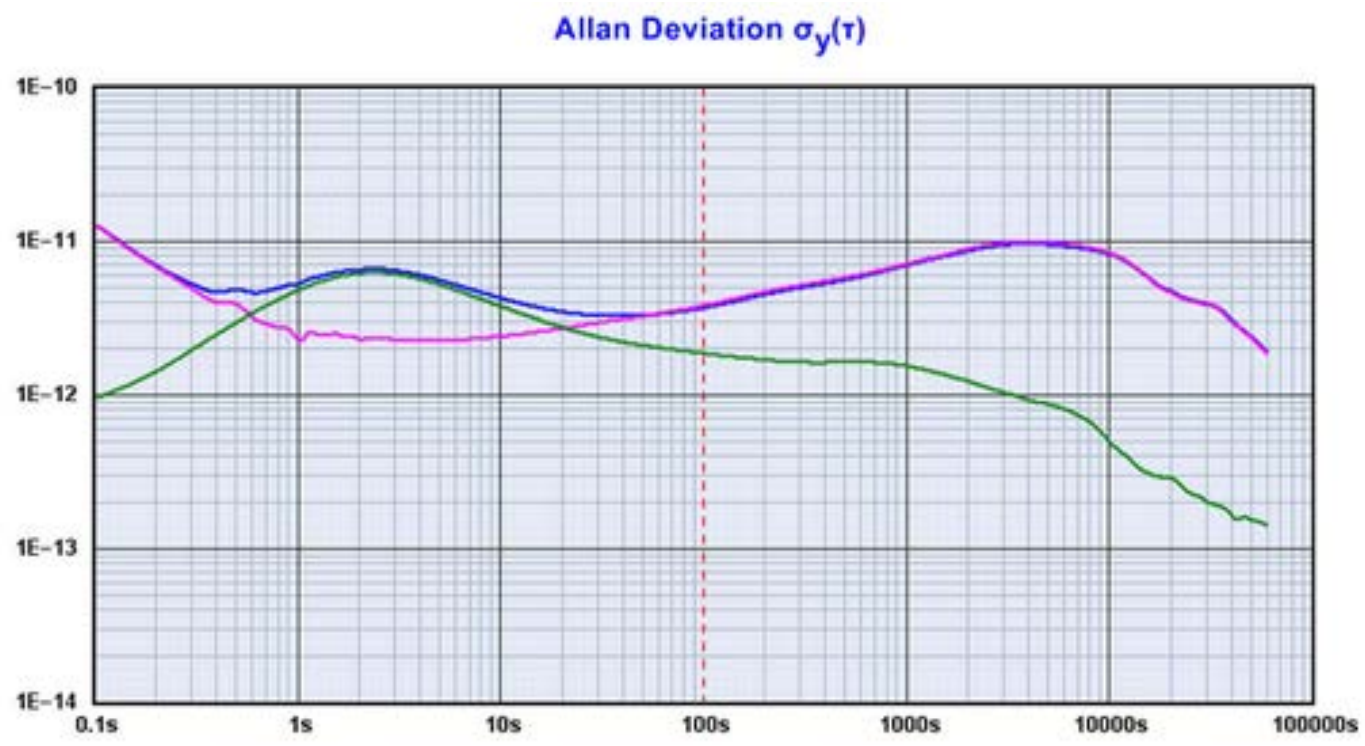

Figure 7. The variation trend of Allan Deviation.

Table 1. The comparison between measured $\sigma_{y}$ and needed $\sigma_{v}$.

\begin{tabular}{|c|c|c|c|c|c|c|c|c|}
\hline $\begin{array}{c}\text { Time } \\
\text { (second) }\end{array}$ & 1 & 2 & 4 & 8 & 10 & 20 & 40 & 80 \\
\hline $\begin{array}{c}\text { The } \\
\text { measured } \sigma_{v}\end{array}$ & $2.87 \mathrm{E}^{-11}$ & $1.95 \mathrm{E}-11$ & $1.43 \mathrm{E}-11$ & $1.01 \mathrm{E}-11$ & $8.12 \mathrm{E}-12$ & 7.17E-12 & $5.73 \mathrm{E}-12$ & $4.99 \mathrm{E}-12$ \\
\hline $\begin{array}{c}\text { The } \\
\text { needed } \sigma_{v}\end{array}$ & $2.65 \mathrm{E}-9$ & $1.32 \mathrm{E}-9$ & $6.63 \mathrm{E}^{-10}$ & $3.31 \mathrm{E}^{-10}$ & $2.65 \mathrm{E}^{-10}$ & $1.32 \mathrm{E}-10$ & $6.63 \mathrm{E}^{-}-11$ & $3.31 \mathrm{E}-11$ \\
\hline Time (second) & 100 & 200 & & & 800 & 1000 & 2000 & 4000 \\
\hline $\begin{array}{c}\text { The } \\
\text { measured } \sigma_{v}\end{array}$ & $4.85 \mathrm{E}-12$ & $5.00 \mathrm{E}-12$ & & -12 & $8.22 \mathrm{E}-12$ & $9.30 \mathrm{E}-12$ & $1.41 \mathrm{E}-11$ & $1.99 \mathrm{E}-11$ \\
\hline $\begin{array}{c}\text { The } \\
\text { needed } \sigma_{v}\end{array}$ & $2.65 \mathrm{E}-11$ & $1.32 \mathrm{E}-11$ & & -12 & $3.31 \mathrm{E}-12$ & $2.65 \mathrm{E}-12$ & $1.32 \mathrm{E}-12$ & $6.63 \mathrm{E}-13$ \\
\hline
\end{tabular}

GPSDO also provides a second pulse signal and synchronization signal for ADC chip.

\section{GPS Timing Strategy}

To ensure that interference signals from different sub-stations are received at the same time the signal will be added time scale using computer's system time. So system time of computers in different sub-stations must be consistent. The GPS timing network receives GPS signal and grant GPS time to computers through LAN.

\subsection{GPS Timing Network}

GPS timing network consists of GPS antenna, NTP (Network Time Protocol) server, router and computers which need GPS time as Figure 8.

GPS antenna receives GPS signal and transmit it to NTP server. NTP server will generate standard time according to NTP time protocol and grant the time to computers through router.

\subsection{GPS Timing Interval Determined}

There are two parameters can be set on the bottom of the client interface of NTP server as Figure 9. The parameter on left side is IP address of NTP server. To ensure NTP server grant GPS time to computers in LAN successfully, computers must in the same IP address segment with NTP server. The parameter on the right side is 


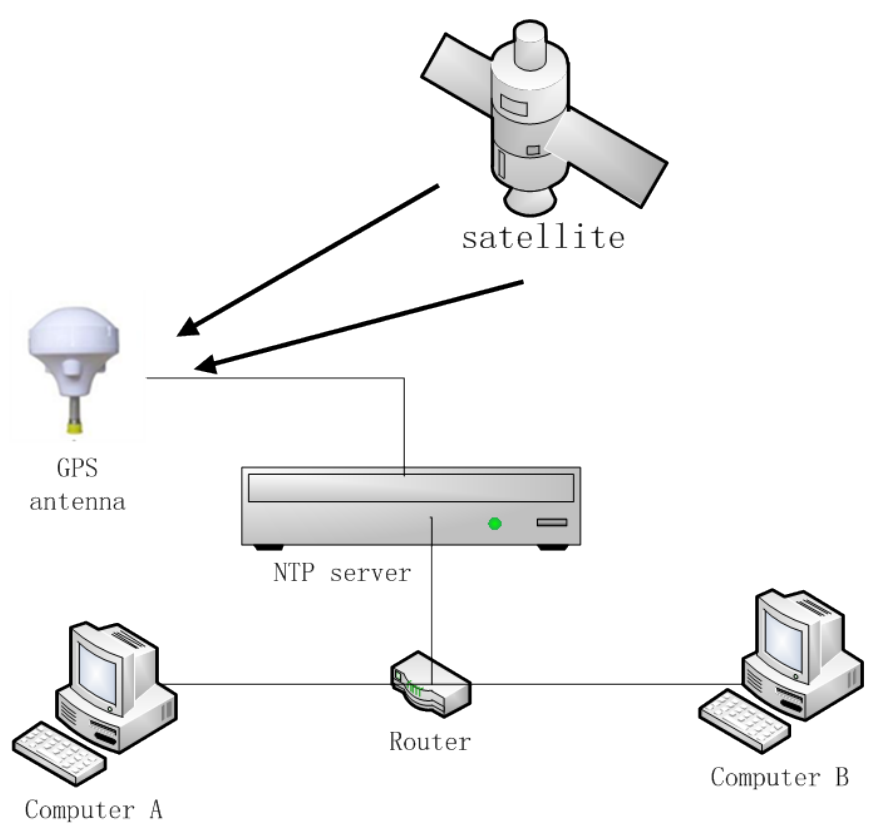

Figure 8. Timing network of GPS.

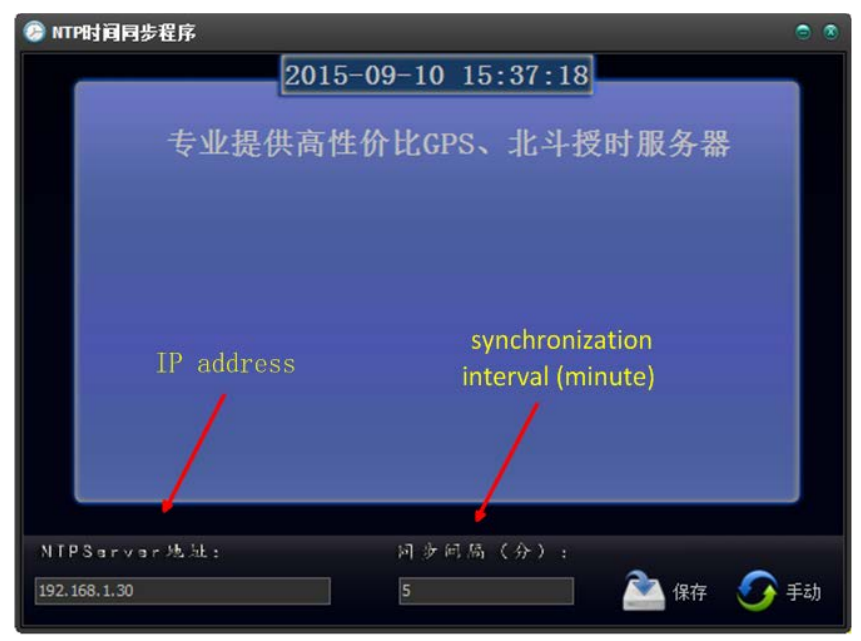

Figure 9. The client interface of NTP server.

synchronization interval (minute), it determines the frequency of NTP server grants GPS time to computers. The system time of computers occurs deviation in different time because of variety of computer hardware and usage situations etc. In order to maximize the use of resource, it is necessary to find a way to determine the time of computer system time deviation occurs.

It is convenient to get and store two computers' system time (the following is called "computer A" and "computer B" and they can be considered as in two sub-stations) through programming in LabVIEW (graphical programming platform). The two computers transmit their system time to the third computer (computer $\mathrm{C}$ ) and the time of system time deviation occurs will be find after analyzing the system time data which stored in computer C.

The task of program in computer A and B is obtain system time and transmit the data to computer C, the program is in Figure 10. The task of program in computer $C$ is receive and store the computer system time data come from computer A and B. The program is in Figures 11-13 are program flow chart of program in computer $\mathrm{A}, \mathrm{B}$ and $\mathrm{C}$ respectively. 


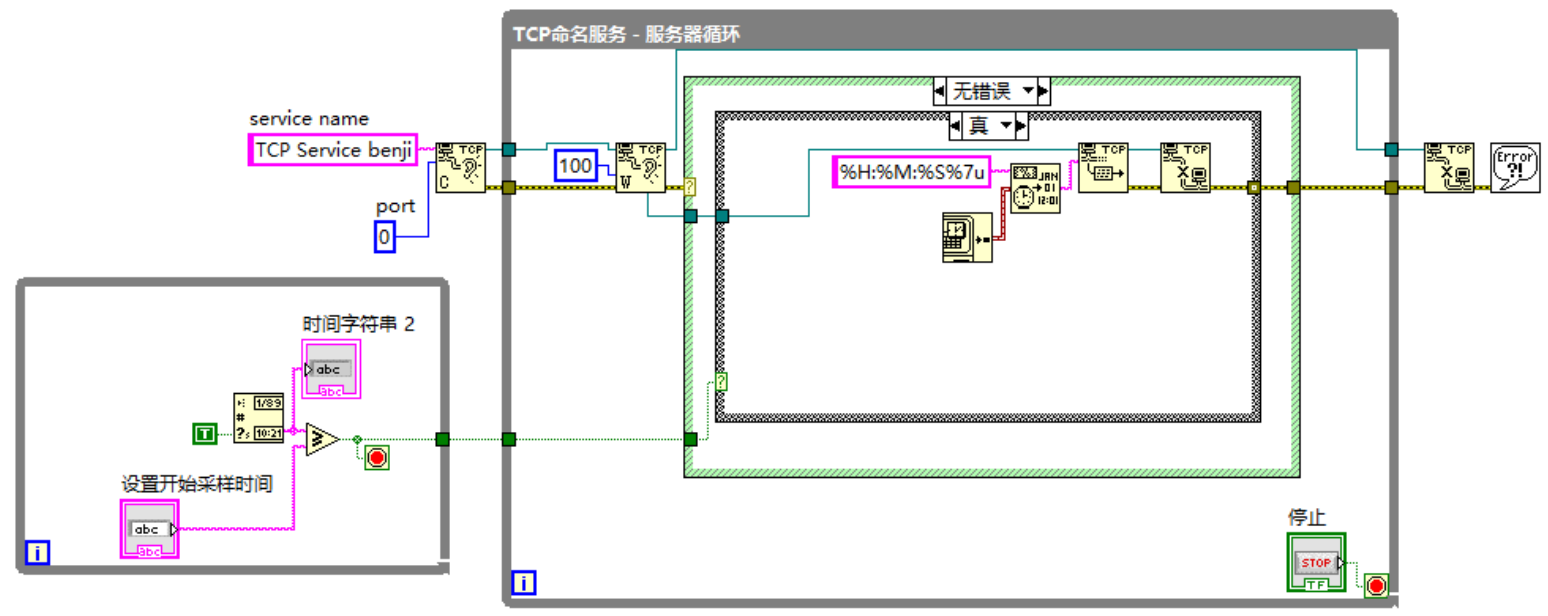

Figure 10. The program in computer A and B.

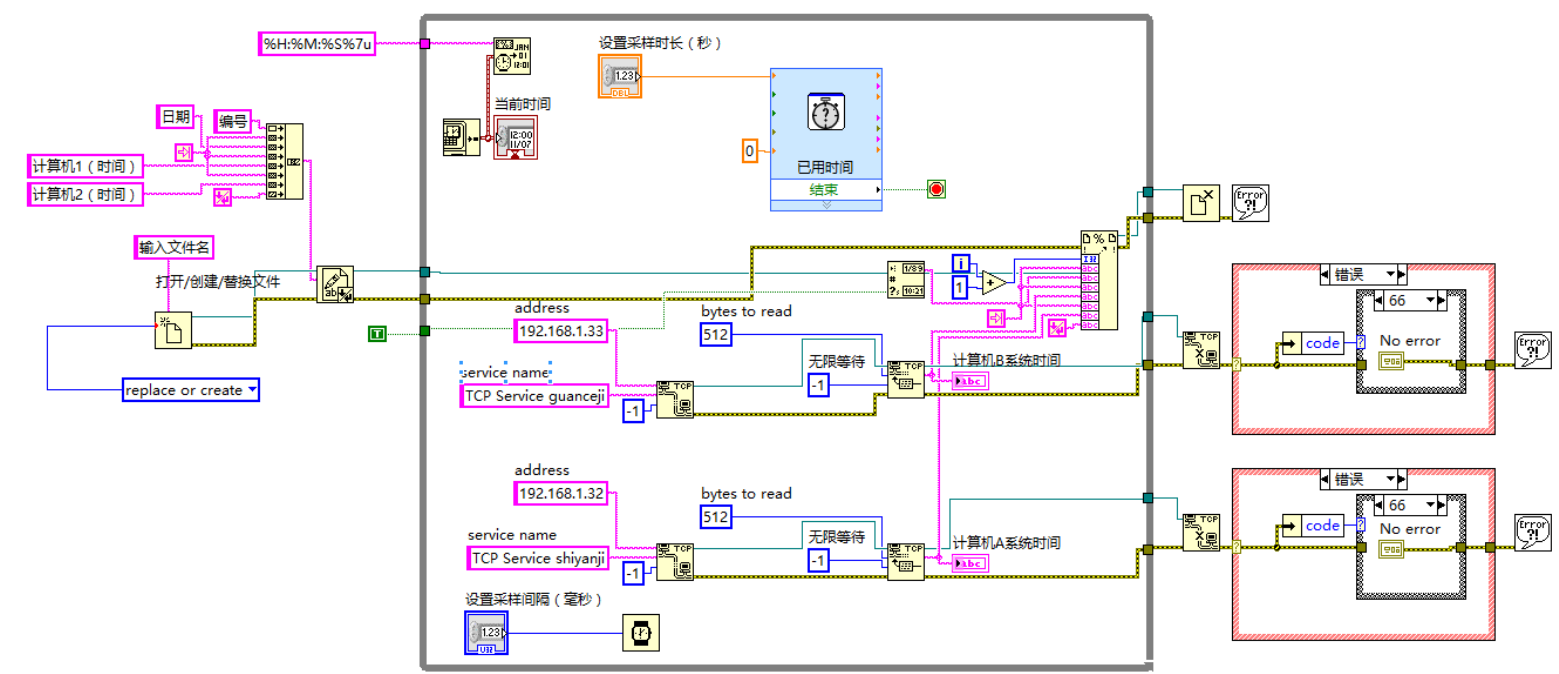

Figure 11. The program in computer $\mathrm{C}$.

Figure 14 is the interface of program in computer C. The "current time" is the system time of computer C, the "system time of computer A" and "system time of computer B" are the system time data transmitted from computer A and B respectively. There are two parameters can be set on the program interface. The "acquisition time (s)" is the time span of computer C obtains system time from computer A and B; the "sampling duration (ms)" is the time interval of computer C receives two adjacent data. If the "acquisition time (s)" is 7200 and "sampling duration (ms)" is 1000 as set in the Figure 14 means computer C will receives one system time data per second from computer A and B respectively, and the program will stop automatically 7200 seconds later. So computer C will receive 7200 system time data from computer A and B respectively in this situation and the next step is analyzing these data to find the time of computers' system time deviation occurs.

\section{Experiment and Conclusions}

Before the experiment started, all computers and NTP server form a local area network to ensure data can be transmit around all equipments. Firstly NTP server grants the GPS time to computer A and B to make sure system time of the two computers are same at the beginning. The parameters of "acquisition time (s)" and "sampling duration (ms)” are set to 6874 and 1000 respectively. Computer A and B send one system time data per second to computer $\mathrm{C}$ and the program will stop 6874 seconds later. After computer $\mathrm{C}$ has obtained all system time data of computer A and B, make system time data of computer A minus system time data of computer B, 


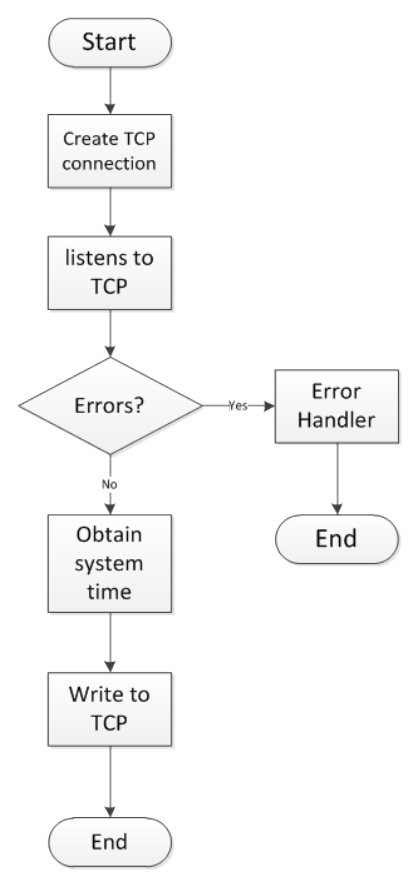

Figure 12. Program flow chart of computer A and B.

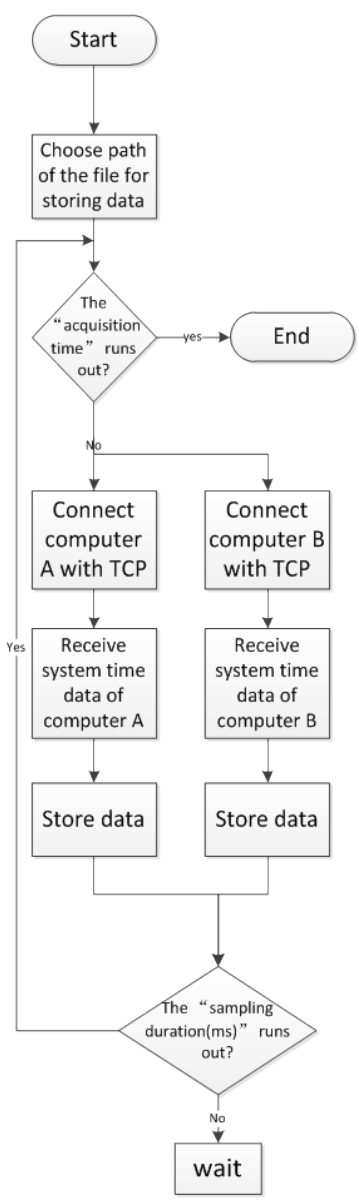

Figure 13. Program flow chart of computer C. 


\section{J. Q. Liu et al.}

and the variation trend of the deviation between system time of the two computers can be graphed through linear fit as Figure 15. It can be found that the deviation of the two computers' system time is zero at beginning, but it grows linearly with time flows. At the second of 4292, the deviation grows to nearly $0.1 \mathrm{~s}$. To maintain the deviation of the two computers' system time within $0.1 \mathrm{~s}$ (it satisfies the demand of observation using the antenna array), NTP server should grant GPS time to the two computers within every 4292 seconds.

To verify the effectiveness of this method, another experiment was performed. The parameter "synchronization interval (minute)" of NTP server was set to 60 (3600 s) to ensure the resource of all system were not wasted. The variation trend of the two computers' time deviation is described as Figure 16, and it can be found the deviation always within $0.1 \mathrm{~s}$. The time-frequency system and timing strategy proposed by this paper are used in the antenna array which has two sub-stations, and it is worthy to mention that the method also effective when the antenna array expanded to several sub-stations more than two.

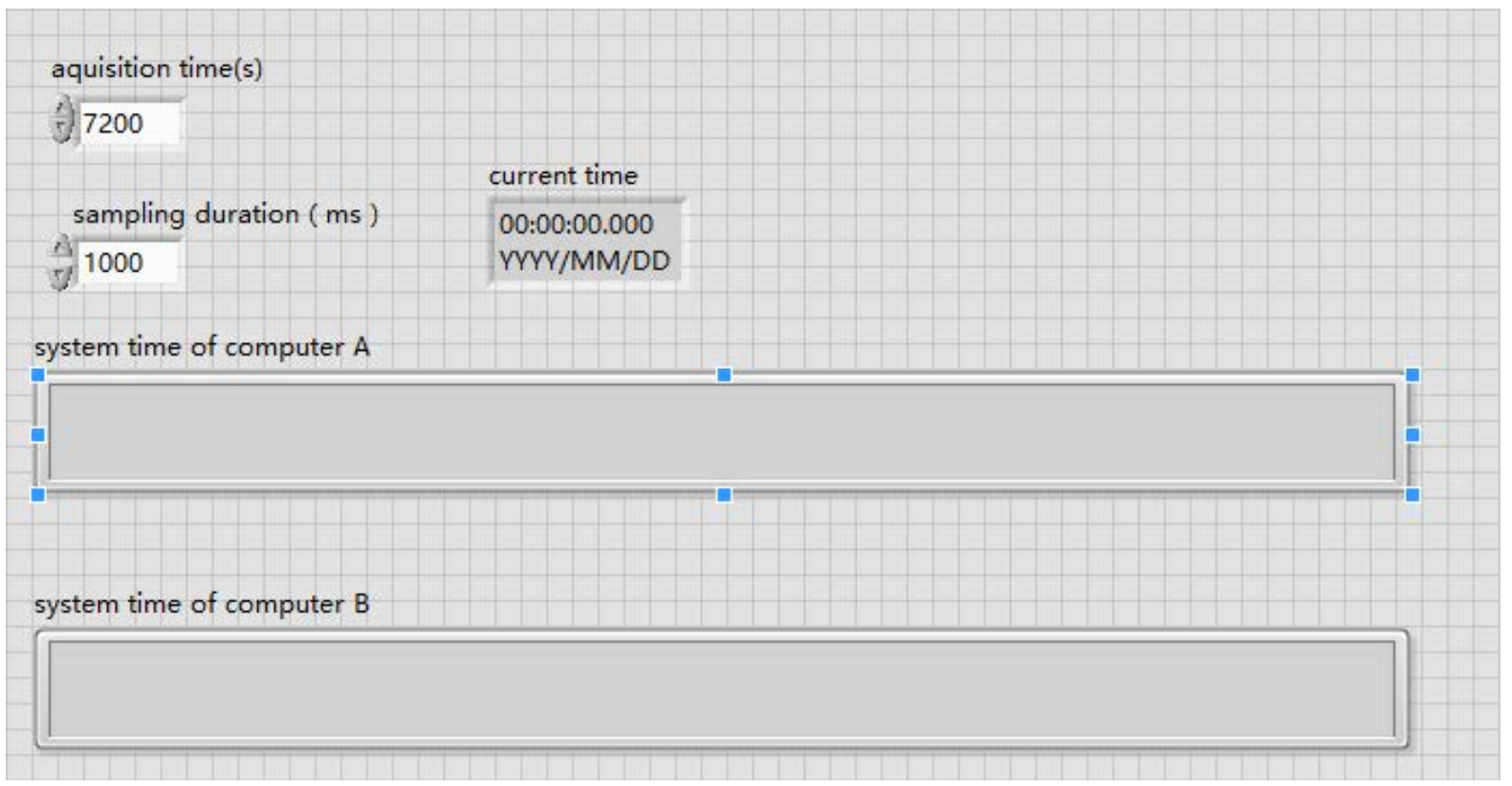

Figure 14. The interface of program in computer C.

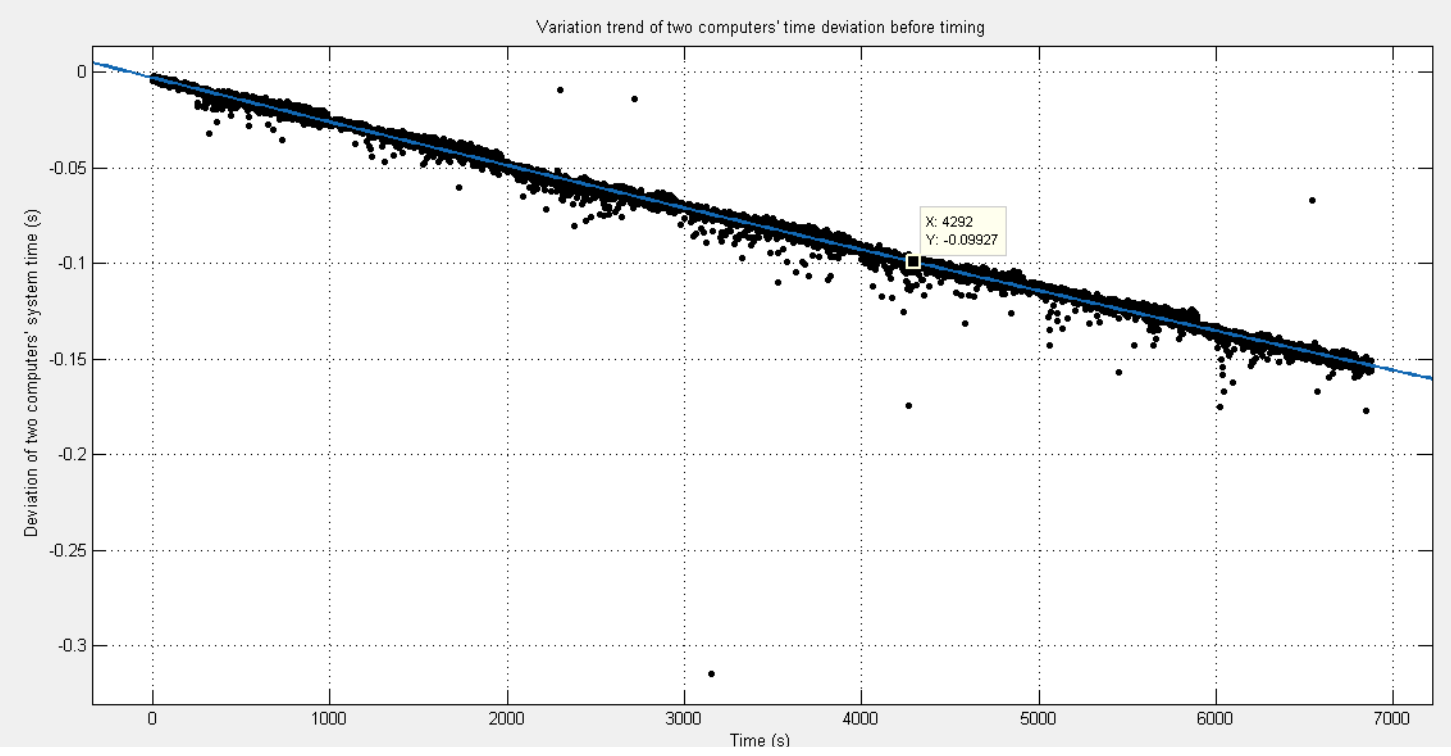

Figure 15. Variation trend of two computers' time deviation before timing. 


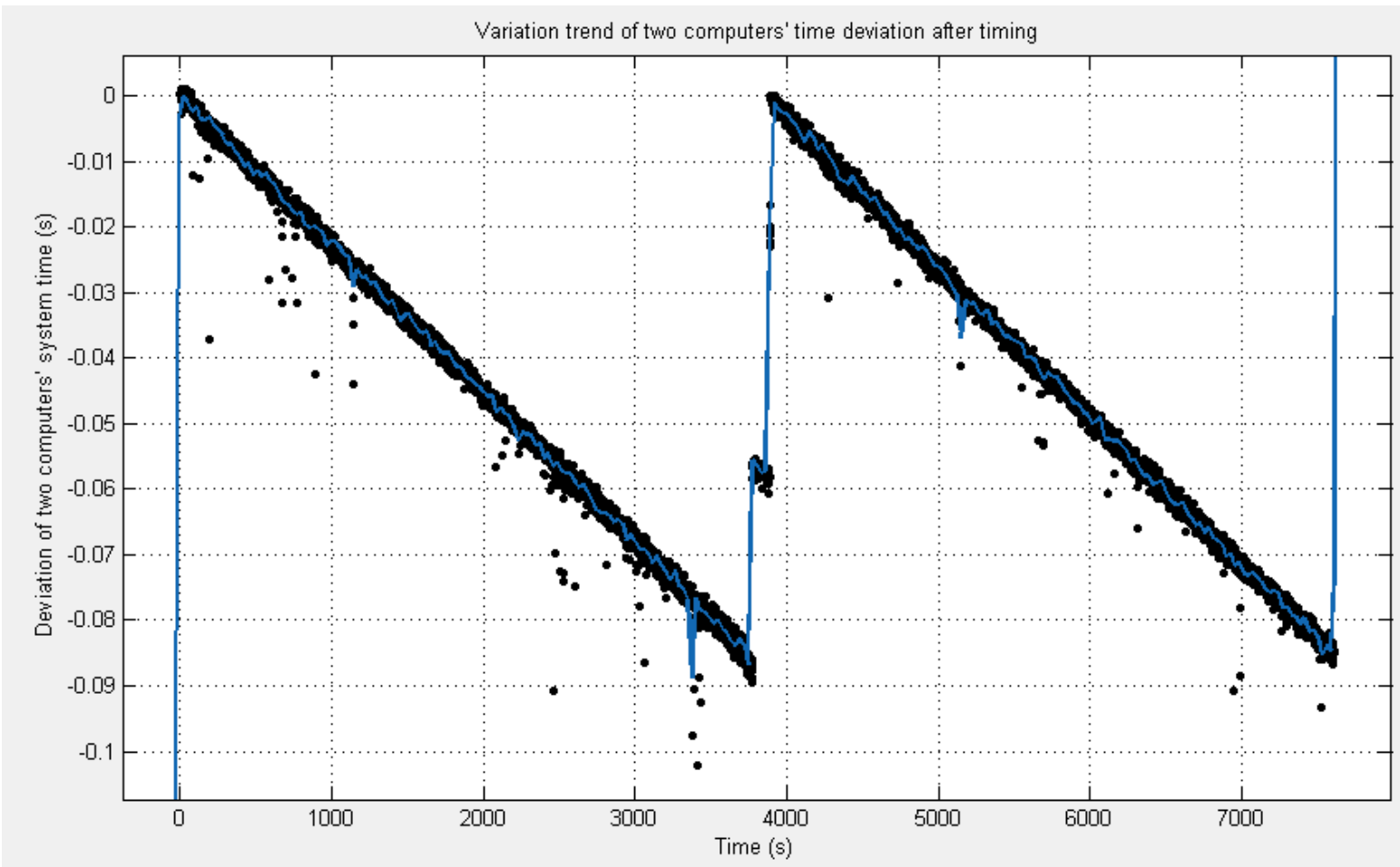

Figure 16. Variation trend of two computers' time deviation after timing.

\section{Acknowledgements}

This work is sponsored by Youth Fund of National Natural Science Foundation of China (11303094), National Natural Science Foundation Astronomy joint fund of China (U1431113), applied basic research program and project of Yunnan province of China (2015FB189) and Western Light A class talent program of China.

\section{References}

[1] Romeney, J.D. (1999) 4. Cross Correlators. Synthesis Imaging in Radio Astronomy II, Edited by TAYOR GB, CARILLI CL , PERLEY R A, ASP Conference Series, 180.

[2] Oberoi, D. and Kasper, J.C. (2004) LOFAR: The Potential for Solar and Space Weather Studies. Planetary and Space Science, 52, 1415-1421. http://dx.doi.org/10.1016/j.pss.2004.09.009

[3] Lantos, P. (1998) Low Frequency Observations of the Quiet Sun: A Review. Solar Physics with Radio Observations. Proceedings of Nobeyama SymposiuNRO Report 479.

[4] Van Trees, H.L. (2008) Detection, Estimation, and Modulation Theory, Optimum Array Processing (Part IV). Tsinghua University Press, Beijing.

[5] Pober, J.C., et al. (2013) The Baryon Acoustic Oscillation Broadband Androad-Beam Array: Design Overview and Sensitivity Forecasts. The Astronomical Journal, 145, 65. http://dx.doi.org/10.1088/0004-6256/145/3/65

[6] Cohen, A.S., et al. (2007) The VLA Low-Frequency Sky Survey. The Astronomical Journal, 134, 1245-1262. http://dx.doi.org/10.1086/520719

[7] Bernardi, G., et al. (2013) A 189 MHz, 2400 deg2 Polarization Survey with Themurchison Widefield Array 32-Element Prototype. The Astrophysical Journal, 771, 105. http://dx.doi.org/10.1088/0004-637X/771/2/105

[8] Evaluating the AD6642/AD6657 Analog-to-Digital Converters.

[9] High Speed Converter Evaluation Platform HSC-ADC-EVALC.

[10] Qian, Z.H. and Li, J.L. (2012) Application of Very Long Base Line Interferometry in Deep Space Exploration. China Science and Technology Press, Beijing. 